\title{
Perspectiva actual sobre el uso de tecnologías de la información y la comunicación en personas con trastorno del espectro autista: Sistematización de experiencias
}

\author{
Current perspective on the use of information and communication technologies in people \\ with autism spectrum disorder: Systematization of experiences
}

\author{
Carmen del Pilar Gallardo Montes ${ }^{1}$ \\ https://orcid.org/0000-0002-6510-2425 \\ María Jesús Caurcel Cara ${ }^{2}$ \\ https://orcid.org/0000-0002-2169-9100 \\ Antonio Rodríguez Fuentes ${ }^{3}$ \\ https://orcid.org/0000-0002-8036-9902 \\ Universidad de Granada, España
}

Recibido: 25-09-2019

Aceptado: 09-12-2019

\section{Cita Recomendada}

Gallardo, C., Caurcel, M. \& Rodríguez, A. (2019).Perspectiva actual sobre el uso de tecnologías de la información y comunicación en personas con trastorno del espectro autista: Sistematización de experiencias. Hamut'ay, 6(3), 50-61. http://dx.doi.org/10.21503/hamu.v6i3.1846

\section{Resumen}

Este artículo sigue un enfoque cualitativo y está centrado en el método científico denominado "sistematización de experiencias”. Tuvo como objetivo analizar la influencia de las Tecnologías de la Información y la Comunicación (TIC) en la sociedad actual y cómo estas herramientas digitales influyen en el ámbito educativo. Se ha indagado en los efectos sobre personas con necesidades especiales, más concretamente para personas con Trastorno del Espectro Autista (TEA). Tras realizar la revisión bibliográfica pertinente -44 documentos- sobre bases de datos consolidadas (WOS, Scopus, Dialnet, Latindex, SciELO, Redalyc, Google Scholar, Eric...) y poniendo especial interés en aquellos investigadores especializados en el ámbito de la educación especial se han podido conocer los beneficios que tienen las TIC en la terapia/intervención psicopedagógica con personas con este trastorno. Se revisaron 10 artículos de investigación, cuyas experiencias exitosas centradas en el uso de materiales digitales (Tablet, Apps, realidad aumentada...), las cuales ofrecen como consecuencia beneficios y mejoras en cuanto a la comunicación, lenguaje, habilidades sociales y emociones. De igual modo, los resultados favorables derivados de la intervención no serían posibles si los profesionales no contasen con la formación adecuada en TIC y en recursos de este tipo. De acuerdo con esto, para una

\footnotetext{
1 Doctorando en el Programa de Ciencias de la Educación en la Universidad de Granada. Máster en Intervención Psicopedagógica por la UGR y Graduada en Pedagogía por la UGR. Miembro del grupo de investigación "Investigación en Comunicación Educativa (ICE)". E-mail: carmengallardo@correo.ugr.es

2 Profesora Contratada Doctora. Dpto. Psicología Evolutiva y de la Educación de la UGR, España. Miembro del grupo de investigación "Atención psicológica y educativa a la diversidad (HUM846)". Participación en proyectos de I+D+I. E-mail: caurcel@ugr.es

3 Profesor Titular de Universidad. Dpto. Didáctica y Organización Escolar de la UGR, España. Miembro del Grupo de Investigación "Investigación en Comunicación Educativa (ICE)” y del "Grupo de Investigación en el Campo de la Discapacidad (GICD)”. Investigador en proyectos nacionales e internacionales. E-mail: arfuente@ugr.es
} 
formación de calidad, será crucial, que las personas con TEA se beneficien de las posibilidades de este tipo de herramientas y materiales digitales. Para concluir, las TIC se presentan como una opción innovadora y motivadora para las personas con este trastorno, ayudando al desarrollo de habilidades en déficit.

Palabras Clave: TIC, educación especial, Trastorno del Espectro Autista, sistematización de experiencias..

\section{Abstract}

This article follows a qualitative design and it's focused on the scientific method called "systematization of experiences". Its purpose was to analyze the impact of Information and Communication Technologies (ICTs) in today's society and how these digital tools influence on the educational field. Their effects on people with special needs have been examined, more specifically on people with Autism Spectrum Disorder (ASD). After having conducted a proper bibliographic review 44 documents- on consolidated databases (WOS, Scopus, Dialnet, Latindex, SciELO, Redalyc, Google Scholar, Eric ...) and paying special attention to those researchers specialized in the field of special education, we have been able to know the benefits of ICTs in psycho-pedagogical therapy and intervention with people with this disorder. Ten research articles were reviewed, whose successful experiences focused on the use of digital materials (Tablets, apps, augmented reality ...), which had as a consequence, benefits and improvements in communication, language, social skills and emotions. Likewise, the favorable results derived from the intervention, would not be possible if the professionals did not have an adequate training in ICTs and resources of this kind. According to this, to provide quality training, it's essential that people with ASD benefit from the possibilities that this type of digital tools and materials offer. To conclude, ICTs are presented as an innovative and motivating option for people with this disorder, helping them to foster underdeveloped skills.

Keywords: ICTs, special education, Autism Spectrum Disorder, systematization of experiences.

\section{INTRODUCCIÓN}

Vivimos y convivimos actualmente en una sociedad en la que el empleo de Tecnologías de la Información y Comunicación (TIC) forma parte del quehacer diario, dándoles uso en ámbitos diversos, como el empresarial, el educativo, el cultural o el del entretenimiento. Según García-Valcárcel (2003, p. 42) las TIC son "el conjunto de tecnologías (ordenadores, consolas de videojuegos, robótica, tabletas digitales, Smartphone, software educativo y demás), que permiten la adquisición, producción, almacenamiento, tratamiento, comunicación y presentación de información en forma de voz, imágenes, y otros”. Dado que las
TIC ofrecen herramientas para el acceso a la información, su uso es continuado y, por ello, favorece el desarrollo y transformación sociocultural.

La Sociedad del Conocimiento en la que nos encontramos inmersos se caracteriza "por la vertiginosa evolución tecnológica y por el incremento exponencial en los niveles de información generada y difundida a través de las TIC. Este auge se ha convertido en una parte relevante en la vida de las personas" (Pérez et al., 2018, p. 452).

Flórez et al. (2017) comentan, a su vez, las nuevas formas de comunicación y de producir información procedente del desarrollo tecnológico y, 
cómo éstas indicen en el quehacer diario de nuestra sociedad. De esta forma, las TIC han incidido de manera sustancial en el día a día, desarrollándose formas más innovadoras para comunicarse y aprender. Así, autores como Grande, Cañón \& Cantón (2016) resaltan que características de las TIC como la instantaneidad, la interactividad o la interconexión, son los aspectos por los que han impactado a nivel social y han cobrado protagonismo. Todo ello favorece el intercambio de información y el desarrollo de nuevos espacios de aprendizaje e interacción.

El impacto progresivo que han tenido dichas tecnologías en diversos espacios de la sociedad ha supuesto que ámbitos como el educativo se enriquezca de ellas, ofreciendo un amplio abanico de posibilidades a docentes y discentes. De hecho, como señala Rojas (2017, p. 2), "a menudo se habla de la implementación de las TIC como una necesidad primordial en las escuelas". Como suma a lo anterior, Guzmán, Putrino, Martínez \& Quiroz (2017) exponen que es necesario que existan investigaciones que evidencien experiencias con resultados favorables sobre el uso de dispositivos tecnológicos en personas con Trastorno del Espectro Autista.

En este sentido, el Trastorno del Espectro Autista cuenta con una trayectoria profunda de investigación, siendo Kanner (1943) el precursor en indagar acerca de la sintomatología de las personas que presentaban este trastorno. Centró su investigación en un grupo de niños que mostraban una serie de comportamientos y características similares, lo que, al día de hoy, ha quedado definido como TEA.

Más concretamente, el Manual diagnóstico y estadístico de los trastornos mentales (DSM-V) en su quinta edición define este trastorno como un conjunto de "deficiencias persistentes en la comunicación social en la interacción social en diversos contextos $[\ldots]$ presentándose patrones restrictivos de comportamiento, intereses o actividades" (APA, 2014, p. 50; Guzmán et al., 2017).

Dicho trastorno se muestra de forma continuada y suele iniciarse antes de los tres ańos de edad. A su vez, determinadas funciones cerebrales superiores se ven afectadas, como el lenguaje o la inteligencia, dando lugar a distintos niveles de afectación.

Son variados los especialistas que tratan a diario con personas con esta condición, como psicólogos, pedagogos, maestros de educación especial, logopedas o médicos. Este equipo multidisciplinar se centra desde edades tempranas en diagnosticar y atender las necesidades del menor, abordando cada ámbito en déficit y trabajando conjuntamente en su desarrollo.

De esta forma $y$, partiendo de las necesidades de la persona con TEA, la terapia psicopedagógica va a seguir unos objetivos concretos. En esta línea, las TIC cobran gran relevancia (Terrazas, Sánchez \& Becerra, 2016; Jiménez, Serrano \& Prendes, 2017), favoreciendo el desarrollo y aprendizaje en multitud de áreas en déficit, como la comunicación, el lenguaje, las emociones, o las habilidades sociales.

\section{Relevancia de las TIC en el ámbito educativo}

La utilización de herramientas tecnológicas es una realidad más que aceptada en nuestro día a día, de la que, por fortuna, el ámbito educativo se nutre de multitud de materiales y recursos específicos en la formación del alumnado, los cuales resultan beneficiosos y motivadores para los estudiantes. En cuanto a las TIC, García-Valcárcel, Basilotta \& López (2014) destacan que estas herramientas despiertan el interés del alumnado con necesidades especiales, dado que favorecen su autonomía y se presentan como una opción alentadora, adecuándose al ritmo y nivel del estudiante.

García, Garrote \& Jiménez (2016, p. 143) recogen las características principales que las TIC ofrecen en el aula: a) Creación de elementos más flexibles para el aprendizaje. b) Eliminación de las barreras espacio-temporales entre el profesor y el estudiante. c) Potenciar los escenarios y entornos interactivos. d) Favorecer el aprendizaje autónomo, colaborativo y en grupo. e) Obtener gran cantidad de información. f) Ser fuente de comunicación.

El uso de TIC tanto en la educación formal como en la no formal ha contado con una aceptación 
progresiva, estando su utilización más consolidada actualmente. En esta línea conviene resaltar que la tecnología actualmente se usa como medio para conseguir un objetivo educativo, no de forma aislada (Rodríguez de Dios \& Igartua, 2015; Zapata-Ros, 2015). La finalidad del uso de las TIC residiría en hallar un método de enseñanza y aprendizaje más alentador y acorde a la realidad y exigencias de la Sociedad del Conocimiento.

El uso de TIC en el aula como hemos dicho antes, no es un hecho aislado, pero no debe hacerse a la ligera, ya que su utilización no alcanzará el éxito pedagógico deseado por parte de la comunidad educativa sin un análisis profundo de las debilidades y fortalezas que se ofrecen.

Para ello, son varios los autores que han profundizado en conocer en cómo el uso de tecnologías favorece el aprendizaje y si a su vez, los docentes presentan los conocimientos necesarios para hacer que se cumplan las expectativas deseadas sobre el alumnado. Desde esta perspectiva, Sánchez, Olmos \& García (2017) en su estudio han planteado como objetivo conocer la resistencia al cambio y la compatibilidad sobre el uso de tecnologías móviles en los futuros maestros, arrojando resultados favorables sobre la intención de uso de móviles. De la misma forma, Brazuelo, Gallego \& Cachiero (2017) analizan las actitudes y posibilidades de uso del móvil en profesorado de Educación Secundaria Obligatoria (ESO), aportando datos que manifiestan, por un lado, el desconocimiento de los docentes acerca de cómo incluir dicho dispositivo en el aula, sumado a un porcentaje significativo que presenta interés por aprender a incluirlo en su labor docente. A su vez, Nicolete et al. (2017) han centrado su investigación en plantear dinámicas para enseñar matemáticas con ayuda de TIC, para de esta forma, alentar al alumnado en sus primeros años de etapa educativa. Por otro lado, Rojas (2017), en su estudio etnográfico sobre la enseñanza de Ciencias Naturales, concluye aportando que la integración de las TIC es necesaria, ya que no únicamente genera competencias vinculadas a lo cognitivo, sino también a nivel actitudinal.

"La incorporación de TIC en el sector de la educación ha permitido configurar nuevos escenarios para las prácticas educativas, que son motivo de investigación y estudio en el campo de la pedagogía” (Caro, Vargas \& Campo, 2015, p. 50). En esta línea, las autoras Toledo \& Llorente (2016) añaden que el ordenador y todos los recursos con los que esta cuenta, se han convertido en aspectos esenciales en lo que la alfabetización de la sociedad concierne.

Dado el interés que despiertan las TIC en el proceso de enseñanza y aprendizaje se hace necesario seguir profundizando en el estudio de sus beneficios en el ámbito académico, sin olvidar la importancia de que toda la comunidad educativa se involucre en hallar resultados exitosos, para que sea posible construir un espacio de innovación y cooperación. De igual modo, es necesario que, dado el interés que los estudiantes de diferentes edades, tienen hacia las TIC, los docentes deben tener una formación idónea y contar con las herramientas adecuadas para transmitir conocimiento.

De tal suerte que es importante resaltar que las TIC han llegado a todos los espacios de la sociedad, dando oportunidades de desarrollo también a estudiantes con necesidades especiales (Rodríguez \& García, 2012), demostrándose de esta forma resultados positivos sobre su uso, lo que requiere garantizar desde la elaboración tanto de hardware como de software de la accesibilidad y usabilidad universales, como puntos de partida (Rodríguez, 2018).

En relación con lo expuesto con anterioridad, este estudio tiene como finalidad el análisis y reflexión de estudios y experiencias centradas en el uso de TIC en personas con TEA. Así, se podrá conocer el contenido de las publicaciones más recientes con resultados favorables en el tratamiento de personas con autismo en relación con el uso de las TIC.

Uso de las TIC en personas con necesidades especiales

La utilización de materiales y herramientas basadas en tecnologías es una realidad de la que tanto la educación formal como la no formal, se nutren cada día. Por ello, al igual que en cualquier inter- 
vención, se precisa de una revisión y una formación acorde a las necesidades del alumno, siendo ésta aún más importante si hablamos de alumnado con necesidades educativas especiales. Como bien detalla el ICT "Competency Standards for Teachers" de la UNESCO (2011), el logro obtenido del uso de TIC dependerá, entre otros factores, de la formación del profesorado en dichos materiales y la capacidad para adecuarlos al contexto. De esta forma, no sería suficiente con dotar a los centros de herramientas basadas en las TIC si el profesorado no cuenta con las destrezas necesarias. De igual modo, la formación recibida tiene que abordar multitud de áreas. Los especialistas en educación especial tienen que contar con conocimientos suficientes que "ayuden a salvar los problemas con los que los alumnos discapacitados se encuentran a diario, barreras que le impiden el aprendizaje." (Toledo \& Llorente, 2016, p. 126). Lo que también depende de la tecnología objeto de empleo, además de la discapacidad por su canal de acceso y adaptabilidad a los usuarios (Rodríguez, 2007; Rodríguez \& Gallego, 2007), lo que puede optimizarse mediante la accesibilidad universal (Rodríguez, 2018).

Como bien detallan Orozco, Tejedor \& Calvo (2017), vincular las TIC a los alumnos con discapacidad supone partir de sus necesidades y respetar sus diferencias para ofrecer una educación de calidad. Si no se partiera de esta premisa, aumentaría la brecha digital, por lo que es importante resaltar la necesidad de una preparación y análisis de necesidades para conseguir solventar las dificultades que pudiesen surgir en la práctica diaria.

Son variados los estudios centrados en la formación del profesorado sobre herramientas TIC (Liu, 2011; Yusof et al., 2014; Altinay \& Altinay, 2015; Vladimirovna \& Sergeevna, 2015). Debido al gran número de herramientas basadas en las TIC que se desarrollan cada día es necesario analizar si los esfuerzos puestos, tanto en materiales como en formación del profesorado, son los deseables. No debe olvidarse que ante todo debe facilitarse el desarrollo integral del alumnado con necesidades especiales, el cual será el protagonista de todo el proceso, y en concreto con Trastorno del Espectro Autista (TEA), sujetos de este trabajo.
De esta forma, el objetivo que sustenta esta sistematización de experiencias es el de hacer un barrido por investigaciones y experiencias exitosas que, habiendo utilizado recursos tecnológicos en personas con TEA, los resultados hayan sido enriquecedores para la población a la que se destinan, mejorando así su desarrollo y calidad de vida en el ámbito de la comunicación, el lenguaje, la interacción social o las emociones. Así, se pretende ofrecer un conjunto de experiencias que muestren de qué forma se han implementado las TIC en el aula o en la terapia y en qué aspectos se ha centrado la intervención psicopedagógica desarrollada por parte del especialista en TEA.

\section{Materiales y Métodos}

\section{Participantes}

$\mathrm{Al}$ ser esta una investigación en la que se realiza un análisis y reflexión de estudios y experiencias centradas en el uso de TIC en personas con TEA, se basa en una revisión documental (Tabla 1), por lo tanto, la muestra estuvo conformada por artículos que versan sobre las dos variables mencionadas, considerando como criterios de inclusión que estos sean de los últimos cinco años (2015-2019), salvo algunos pertenecientes a ańos previos, cuyo aporte sea relevante para situar en qué consiste el TEA (antecedentes y manuales). Se han analizado investigaciones recientes, dada la velocidad a la que avanzan los recursos digitales y la rapidez con la que determinadas tecnologías quedan obsoletas. De esta forma, se han presentado resultados actuales en torno al uso de TIC en personas con TEA.

\section{Instrumento}

En la elección del instrumento de análisis, se ha considerado lo referido por López y Fachelli (2015), que las encuestas son unas técnicas de investigación científica cuyo objetivo es conseguir medidas sobre los conceptos que proceden de un problema concreto. En las encuestas hay que señalar debidamente los rasgos y condiciones, y la recogida de datos. Además, con la ayuda de dicha herramienta desarrollada se observan dos supuestos, que validarán el 
estudio: en primer lugar, los ítems proporcionados permitirán responder al objetivo $\mathrm{y}$, en segundo lugar, con esta acción se busca la involucración de los estudiantes (Monje, 2011).

La encuesta está conformada por un total de 58 ítems intercalando preguntas abiertas y cerradas, además de una escala Likert. Estos ítems a su vez, están divididos en tres dimensiones: a) perfil socio-demográfico, b) conocimiento y uso de las TIC y c) motivación, competencias y habilidades gracias a las TIC (Tabla 1).

\section{Tabla 1}

Criterios de inclusión para la sistematización de experiencias

\begin{tabular}{lll}
\hline Tipo de material & Idiomas & $\begin{array}{l}\text { Intérvalo de fe- } \\
\text { chas }\end{array}$ \\
\hline Artículos de revistas & Español & $\begin{array}{l}5 \text { años } \\
(2015-2019)\end{array}$ \\
\hline Libros en formato digital & Inglés & $(20$. \\
\hline Comunicaciones en congresos & & \\
\hline
\end{tabular}

Fuente: Elaboración propia (2019).

Así, se ha accedido a diferentes bases de datos, como Dialnet, Scopus, Latindex, Scielo, Redalyc, Google Scholar, Web of Science, o ERIC (Tabla 2). La revisión de la literatura ha abarcado un total de 44 documentos (Tabla 3), donde se incluyen libros, artículos de revista y comunicaciones presentadas a congresos internacionales. Para contextualizar la presencia de TIC en el ámbito educativo, se han citado 14 trabajos. En relación al uso que se hacen de las TIC en el ámbito de la educación especial, se ha reflexionado en torno a 10 investigaciones. Para concluir con los beneficios, que las personas con TEA tienen en los recursos digitales, se han analizado 10 investigaciones. Para mayor énfasis, se han citado autores que refuerzan los beneficios de las tecnologías en personas con este trastorno, agregando a los anteriores, 6 artículos de investigación. De esta forma, se ha pretendido ofrecer un análisis de lo general a lo específico, que en este caso sería el TEA.

\section{Tabla 2}

Bases de datos consultadas para la sistematización de experiencias

\begin{tabular}{lcc}
\hline Bases & Documentos & $\%$ \\
\hline Dialnet & 18 & 40.91 \\
\hline
\end{tabular}

\begin{tabular}{lcc}
\hline Scopus & 10 & 22.73 \\
\hline Latindex & 4 & 9.09 \\
\hline Redalyc & 3 & 6.82 \\
\hline SciELO & 2 & 4.55 \\
\hline WOS & 1 & 2.27 \\
\hline Google Scholar & 1 & 2.27 \\
\hline Eric & 1 & 2.27 \\
\hline Otras fuentes & 4 & 9.09 \\
\hline
\end{tabular}

Fuente: Elaboración propia (2019).

Tabla 3

Número de documentos revisados para desarrollar el método de sistematización de experiencias

\begin{tabular}{lc}
\hline Bases & $\begin{array}{c}\text { Nro. de } \\
\text { documentos }\end{array}$ \\
\hline Complementarios (antecedentes/ manuales) & 4 \\
\hline Centrados en las TIC en educación & 14 \\
\hline Centrados en las TIC en educación especial & 10 \\
\hline $\begin{array}{l}\text { Centrados en los beneficios de las TIC en } \\
\text { personas con TEA }\end{array}$ & 16 \\
\hline
\end{tabular}

Fuente: Elaboración propia (2019).

Las intervenciones desarrolladas, así como los estudios sobre beneficios de TIC, se enmarcan tanto en el panorama nacional como en el internacional (Figuras 1 y 2), dando lugar a un repertorio de investigaciones que abarcan distintas formas. Las palabras clave utilizadas en la búsqueda han sido: TIC, Discapacidad, Autismo, TEA, Móvil, Apps, Educación, Educación Especial, Profesorado y Formación.

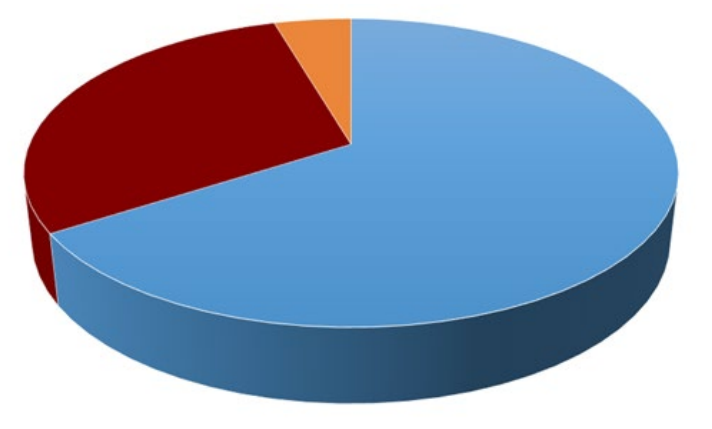

Figura 1. Número de documentos analizados según área territorial.

Fuente: Elaboración propia 
Perspectiva actual sobre el uso de tecnologías de la información y la comunicación en personas con trastorno del espectro autista: Sistematización de experiencias

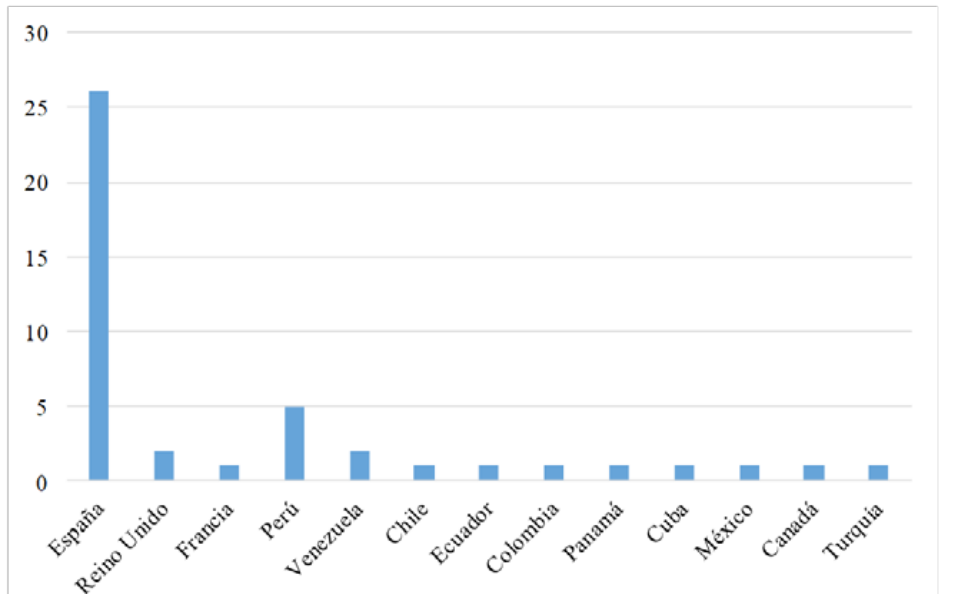

Figura 2. Número de documentos analizados de cada país Fuente: Elaboración propia (2019)

\section{Tipo y diseño de estudio}

La realización de esta investigación sigue un tipo de enfoque cualitativo, atendiendo de esta forma al método científico denominado "sistematización de experiencias". Expósito \& González (2017) plantean la sistematización de experiencias como "un proceso de reflexión e interpretación crítica de la práctica y desde la práctica, que se realiza con base en la reconstrucción y ordenamiento de los factores objetivos y subjetivos que han intervenido en esa experiencia" (p. 1). Todo este proceso implica exponer los hechos que han dado lugar a determinadas experiencias, con la finalidad de comprender qué factores han tenido lugar y de qué forma se relacionan, contribuyendo de esta forma a la construcción de nuevo conocimiento. En consecuencia, la reflexión de los diferentes estudios centrados en el uso de TIC en personas con TEA ha dado forma a este trabajo.

\section{Procedimiento}

El estudio se ha realizado atendiendo a las palabras clave citadas anteriormente y realizando secuencialmente búsquedas de las mismas en diversas bases de datos contrastadas científicamente. De igual modo, los documentos seleccionados finalmente han sido aquellos más vinculados a la temática que nos concierne y que presentan experiencias gratificantes sobre el uso de TIC en la educación de personas con TEA (Figura 3).

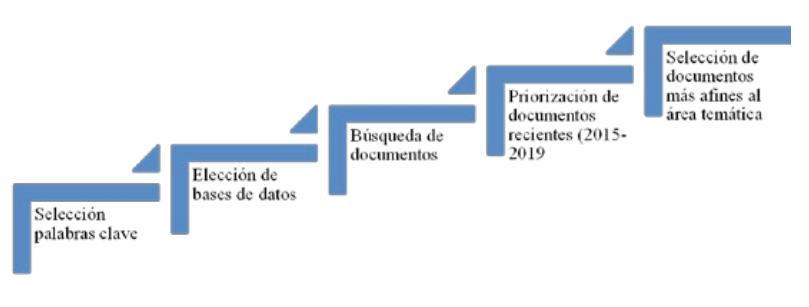

Figura 3. Proceso para el análisis de la información. Fuente: Elaboración propia (2019)

Partiendo de la gran diversidad de investigaciones centradas en dichas palabras clave, se han seleccionado las más relevantes, siendo éstas las que presentan recursos más innovadores y enriquecedores tanto para la comunidad investigadora como para los docentes pertenecientes al ámbito de la educación especial. Del mismo modo, se han tenido en cuenta aquellos autores con resultados exitosos y experiencias que exponían los pros y contras en la intervención propuesta y desarrollada. De esta forma, la perspectiva sobre los beneficios de las TIC en personas con TEA es más amplia.

\section{Resultados}

En base a la revisión realizada y a las revistas encontradas, se ha podido apreciar como mucha de la producción centrada en TIC y su empleo por personas con TEA se encuentra ubicada en revistas españolas, seguida de aquellas pertenecientes a Latinoamérica. En este sentido, se procederá a plantear aquellos trabajos cuyo eje principal ha sido revelar intervenciones exitosas a partir de herramientas digitales. De esta forma se evidenciarían los beneficios que tienen las TIC en la terapia destinada a personas con este trastorno. A su vez, se muestran cómo se han tenido lugar las diferentes experiencias y la forma de llevarse a cabo por parte de profesionales en TEA, sirviendo estas de precedente para posteriores intervenciones psicopedagógicas promovidas por especialistas en la materia.

Como ya se citaba con anterioridad, el TEA se presenta como un trastorno con diferentes grados de afectación en ámbitos vinculados a la comunicación y a la interacción social. De este modo, la intervención psicopedagógica se centra en de- 
sarrollar y promover todo lo que estos ámbitos abarcan. En esta línea, las TIC ofrecen un abanico de posibilidades para fortalecer habilidades en déficit, donde los apoyos visuales y sonoros cobran relevancia. Dichos recursos digitales son alentadores para los niños con autismo (Pérez, 2000), dado que favorecen la atención, la motivación y disminuyen la frustración de la persona con TEA.

En esta línea, son numerosos los autores que han profundizado en conocer, cómo el uso de herramientas digitales aporta beneficios en el desarrollo integral de la persona con TEA. La integración de TIC tanto en el aula como en la intervención/ terapia que reciben los niños como los adolescentes, lo cual aporta resultados sorprendentes, debido a que se presentan de manera interactiva y llamativa, captando así su atención.

En la revisión bibliográfica realizada por Silva \& Rodríguez (2018) los autores encuentran investigaciones actuales cuyo eje principal son la inclusión de las TIC en el currículo académico, resaltando en este artículo, cómo éstas refuerzan el proceso de enseńanza-aprendizaje de los estudiantes con TEA y otras discapacidades.

Adicionalmente al estudio anterior, y más reciente, Castro \& Mallón (2019) presentan un artículo de investigación basado en la revisión bibliográfica de 50 publicaciones sobre el uso de la Tablet en la escuela, en la base de datos Scopus, durante el periodo 2016-2019. La presencia de la Tablet en el aula ha crecido exponencialmente, apareciendo más a menudo en la intervención educativa realizada en personas con diversidad de trastornos, como el TEA, utilizándose sobre todo en aspectos vinculados a la lectoescritura en nińos con esta condición. A su vez, estos autores refuerzan el interés en esta temática por parte de la comunidad científica, ya que el 99\% de los estudios analizados, coinciden en los beneficios obtenidos del uso de la Tablet en la atención a la diversidad.

Lozano \& Merino (2015) realizaron una intervención educativa usando TIC en un centro educativo de Murcia (España), centrada en el desarrollo de emociones. En su artículo, colaboraron tres estudiantes de Educación Secundaria con TEA pertenecientes a un aula específica, los progenitores de los mismos, la maestra de Pedagogía
Terapéutica e investigadores universitarios. La intervención concluye vislumbrando mejoras en el rendimiento de tareas centradas en la comprensión de emociones.

Por su parte, Suárez, Mata \& Peralbo (2015) tras su programa de intervención relacionado con la Teoría de la Mente en un estudio de caso único, encontraron mejorías con relación a las habilidades sociales. El artículo muestra cómo, tras utilizar las aplicaciones "Aprender jugando" y "Second Life", en diferentes sesiones, la atención y motivación del alumno se ha visto reforzada.

En esta línea, el artículo de investigación de Terrazas et al. (2016) se centra en recopilar páginas webs, aplicaciones móviles y programas para ordenador, mediante los cuales las personas con TEA puedan desarrollar diversas habilidades según la necesidad educativa que presenten. Los recursos digitales propuestos se organizan según aspectos comunicativo-lingüísticos, al ámbito social y emocional o el cognitivo. Los autores concluyen apoyando la idea de que las TIC resultan beneficiosas en la intervención de este trastorno, ya que "son una herramienta que, si sabemos cómo, cuándo y para qué utilizarlas potencian significativamente el desarrollo de las personas con TEA. Además, ciertos elementos que nos ofrecen estas tecnologías no las podemos encontrar en otros recursos materiales" (p. 133). En este sentido, Jiménez et al. (2017) realizan un estudio sobre el uso de la Tablet y Apps en el desarrollo de la comunicación y el lenguaje. En este artículo toman como referencia aplicaciones móviles adecuadas a las necesidades de un niño con TEA de un centro público de Murcia. Se utilizaron 30 Apps, algunas de ellas son "CPA", "LetMeTalk", "Azahar", "Communicate", "Find me" o "Dime". Los autores concluyen mostrando progresos en aspectos previos al lenguaje y la intención comunicativa del alumno, añadiendo que el uso de la Tablet y de las Apps ha motivado y atraído al niño.

En relación con lo anteriormente citado, García et al. (2016) indagaron sobre cómo las aplicaciones móviles (Apps) destinadas a personas con autismo, ofrecen beneficios en cuanto al desarrollo de habilidades sociales y la estimulación de los sentidos. En su artículo de investigación hacen 
alusión a cómo las TIC favorecen el trabajo de los maestros de Pedagogía Terapéutica, ya que estas herramientas se presentan de manera más visual y auditiva, y, por consiguiente, proporcionan canales de comunicación más accesibles. En este contexto, Romero \& Harari (2017) centraron su estudio en la realidad aumentada como método para el tratamiento en nińos con TEA. El objetivo de su artículo era conocer la predisposición de familias y profesionales en este trastorno ante la utilización de TIC en la intervención con menores. Los resultados indican altos niveles de interés hacia este recurso didáctico y se muestran receptivos ante esta forma de enseñanza-aprendizaje.

Con respecto a lo antes planteado, González et al. (2016) también resaltan los aspectos positivos encontrados en el aula tras el uso de dispositivos móviles. En su intervención detallan que los dispositivos móviles ofrecen un refuerzo inmediato, porque "las variables de la prosodia y el volumen utilizado se puede mantener estable, ya que son previamente ajustados. Estas variables fundamentales en el caso de los alumnos con TEA, son más difíciles de mantener por el profesor" (p. 46). Del mismo modo, en su artículo plantean que "algunos alumnos con TEA muestren desagrado a los rostros humanos y a mantener la mirada con el interlocutor, por lo que los dispositivos móviles les "salvan" de esa incomodidad" (p.46).

Enriqueciéndonos de los avances que las TIC suponen para los nińos con TEA, un uso adecuado de ellas se presenta como un recurso en auge para el aprendizaje. Se puede ver como el desarrollo de la tecnología nos aportan grandes adelantos y permite ampliar un gran abanico de posibilidades en el campo educativo (Silva \& Rodríguez 2018) promoviendo nuevos espacios de trabajo (Terrazas et al. 2016; Castro \& Mallón 2019), ofreciendo recursos que motivan al alumnado con TEA (Pérez, 2000; Suárez et al., 2015; Jiménez et al., 2017, García-Valcárcel et al., 2014) y permitiendo la formación en el momento en el que el usuario lo desee (García-Valcárcel et al., 2014).

Estamos en un momento en el cual, el acceso a internet permite disponer de cientos de materiales diseñados para personas con TEA en un instante. Desde plataformas webs que ofrecen materiales de trabajo y ocio hasta aplicaciones móviles (Apps). Las Apps desarrolladas giran en torno a ámbitos en que los usuarios con este trastorno presentan carencias, como pueden ser la comunicación, el lenguaje, la gestión del tiempo, el conocimiento y gestión de emociones, la autonomía, entre otras. De acuerdo con ello, las Apps destinadas a este colectivo son numerosas, autores como Suárez (2017) revelaban que en el año 2017 ya existían más de 1.300 de estas Apps. La proliferación de las mismas puede comprobarse haciendo una sencilla búsqueda en los catálogos de aplicaciones como "Google Play Store" para Android o en "App Store" en iOS utilizando las palabras clave como "trastornos del espectro autista" "autismo", "autism" o "asperger". No obstante, es primordial saber distinguir aquellas Apps que ayudarán a reforzar aprendizajes previos de aquellas no necesariamente vinculadas a personas con TEA. De esta forma es fundamental contar con la formación necesaria para saber cómo guiar el proceso de enseñanza-aprendizaje hacia resultados exitosos, sabiendo qué Apps serán las adecuadas para intervenir según qué aspecto se considere, teniendo presente el objetivo propuesto en la terapia a desarrollar.

\section{Discusión y Conclusiones}

El uso de recursos digitales, como se ha ido desarrollando a lo largo del presente artículo, es una realidad más que implantada, en el día a día de las personas con necesidades especiales (Escobar et al. 2016; Orozco et al. 2017; Hernández, 2017). De tal suerte, los avances en el ámbito de la educación especial no se quedan atrás, ofreciendo experiencias gratificantes en el desarrollo íntegro de la persona con autismo, abordando intervenciones desde edades tempranas. En este sentido y en base a estudios previos, son varios los autores que coinciden en señalar que las TIC ofrecen una forma innovadora de enseñanza y resulta un recurso didáctico a tener en cuenta (González et al. 2016; Romero \& Harari 2017; Silva \& Rodríguez 2018; Castro \& Mallón 2019). A su vez, las facilidades que conceden las tecnologías (Carrillo \& Pachón, 2011) y la flexibilidad que presentan a la persona TEA (Cabero, 2008; Hernández, 2017) 
ofrecen herramientas que van a promover el proceso de enseñanza-aprendizaje y, por consiguiente, la inclusión educativa (Fernández, Román \& El Homrani, 2017).

Se hace mucho hincapié en la importancia que tiene que el profesional en educación especial cuente con una adecuada formación sobre estrategias de aprendizaje vinculadas al uso de tecnologías. Es bien sabido que, si se desconoce la forma de uso de determinadas herramientas digitales, se dará un uso arbitrario y el desarrollo de la programación establecida no será exitoso. En este sentido autores como Pérez (2000), Lozano et al. (2013) y González et al. (2016) alertan, a su vez, del mal uso que puede derivar la utilización de dispositivos electrónicos si no se hace una praxis responsable y programada de los mismos.

Partiendo de que las TIC despiertan interés en las personas con TEA, conocer cómo relacionarlas con ejercicios diarios, estudiando sus debilidades y fortalezas, así como alternativas, dará lugar a una intervención exitosa y enriquecedora. Experiencias como las de Terrazas et al. (2016), Lozano et al. (2013) o García et al. (2016) describen cómo estas tecnologías potencian el desarrollo de diversidad de habilidades, relacionadas con la comunicación, el lenguaje, el ámbito emocional o el cognitivo. Conociendo al niño o al adulto, y teniendo presente los diferentes recursos tecnológicos, se podrá conocer qué utilizar según el contexto y cómo mantener la atención en el aula o en casa. Hay que respetar los ritmos de aprendizaje de la persona y marcar pautas de trabajo, para que la intervención con el uso de materiales digitales sea lo más fructífera posible. Dado que las TIC se presentan también como un medio de entretenimiento y ocio, el uso responsable de las mismas en personas con autismo, hará que trabajar con ellas sea más fácil. Ante todo, no hay que confundir en qué momento se le ofrece el recurso digital, ya sea Smartphone o Tablet, para que el objetivo perseguido no se pierda de vista. Estos recursos son inclusivos, ya que se presentan de manera flexible y ofrecen atención individualizada, según las necesidades del usuario, ofreciendo más oportunidades de desarrollo y aprendizaje.

Cabe resaltar la escasa investigación centrada en el uso de TIC fuera del ámbito escolar o incluso, en personas más mayores. De cara a investigaciones futuras, sería valioso indagar también cómo se incluyen las TIC más allá de la niñez o la adolescencia. Las utilidades que ofrecen las herramientas digitales son beneficiosas para todos los rangos de edad, ya que la intervención con personas con este trastorno es imprescindible en todas las etapas de la vida. Para ello, conocer qué recursos se usan en diferentes edades será igualmente práctico en el trabajo con personas con TEA.

Además, sería interesante analizar qué recursos tecnológicos se ofrecen a personas con autismo, indagando en las Apps disponibles para Smartphone y Tablet. Debido al gran número de App que, a priori, son encontradas en una búsqueda rápida en Google Play Store, sería interesante elaborar un instrumento válido y fiable para determinar de esta forma cuáles son las más recomendadas para trabajar según el área de trabajo. Conociendo qué ofrece cada App y qué ámbitos se trabaja en cada una de ellas (comunicación, emociones, gestión del tiempo, habilidades instrumentales básicas -desarrollo del lenguaje, matemáticas, aprendizaje de las letras...-, funciones ejecutivas -organización, memoria, atención, razonamiento...- y ocio/entretenimiento") la programación de la intervención será más rápida y accesible.

\section{REFERENCIAS BIBLIOGRÁFICAS}

Altinay A. \& Altinay, Z. (2015). Examination on ICT integration into Special Education Schools for Developing Countries. TOJET: the Turkish Online Journal of Educational Technology, 14(3), 70-72. Recuperado de https://eric. ed.gov/?id=EJ1067709

APA - American Psychiatry Association (2014). Manual de diagnóstico y estadístico de los trastornos mentales (DSM-V), $5^{a}$ edición. Madrid, España: Editorial Médica Panamericana.

Brazuelo, F., Gallego, D. J., \& Cacheiro, M. L. (2017). Los docentes ante la integración educativa del teléfono móvil en el aula. Revista de Educación a Distancia (RED), 52, 1-22. https://doi.org/10.6018/red/52/6

Cabero, J. (2008). TICs para la igualdad: la brecha digital en la discapacidad. Anales de la Universidad Metropolitana, 8(2), 15-43. Recuperado de https://dialnet.unirioja.es/servlet/articulo?codigo $=3622506$ 
Perspectiva actual sobre el uso de tecnologías de la información y la comunicación en personas con trastorno del espectro autista: Sistematización de experiencias

Caro, L.A., Vargas, T. E. \& Campo, J. A. (2015). Estudio preliminar sobre el uso de Tecnologías de Información y Comunicación en instituciones de Educación Superior de la Red Ilumno. Hamut'ay, 2(1), 49-62.

https://doi.org/10.21503/hamu.v2i1.829

Carrillo, E. \& Pachón, C.M. (2011). Creación, diseño e implantación de plataforma e-learning utilizando mundos 3d para los niños con trastorno del espectro autista (TEA). Revista educación y desarrollo social, 1, 70-80. https://doi. org/10.18359/reds.893

Castro, M. \& Mallón, O. (2019). La Tablet en la escuela: Revisión bibliográfica en Scopus. Hamut'ay, 6(1), 124-139. https://doi.org/10.21503/hamu.v6i1.1579

Escobar, J.P., Arroyo, R., Benavente, C., Díaz, R., Garolera, M., Sepúlveda, A., \& Veliz, S. (2016). Requisitos, retos y oportunidades en el contexto del desarrollo de nuevas tecnologías con niños para niños con discapacidad. Revista nacional e internacional de Educación Inclusiva, 9(3), 127-143. Recuperado de https://dialnet.unirioja.es/servlet/articulo?codigo $=5986225$

Expósito, D., \& González, J. (2017). Sistematización de experiencias como método de investigación. Gaceta Médica Espirituana, 19(2), 1-6. Recuperado de http://revgmespirituana.sld.cu/index.php/gme/article/view/1497

Fernández, J.M., Román, P. \& El Homrani, M. (2017). TIC y discapacidad. Conocimiento del profesorado de educación primaria en Andalucía. Aula Abierta, 46, 65-72. https://doi. org/10.17811/rifie.46.2017.65-72

Flórez, M., Aguilar, A.J., Hernández, Y.K., Salazar, J.P., Pinillos-Villamizar, J.A. \& Pérez, C.A. (2017). Sociedad del conocimiento, las TIC y su influencia en la educación. Revista ESPACIOS, 38(35), 39-50. Recuperado de http://www. revistaespacios.com/a17v38n35/17383539.html

García, S., Garrote, D. \& Jiménez, S. (2016). Uso de las TIC en el Trastorno de Espectro Autista: aplicaciones. EDMETIC, Revista de Educación Mediática y TIC, 5(2), 134-157. Recuperado de https://helvia.uco.es/xmlui/handle/10396/14211

https://doi.org/10.21071/edmetic.v5i2.5780

García-Valcárcel, A. (2003). Tecnología Educativa: Implicaciones educativas del desarrollo tecnológico. España, Madrid: Editorial La Muralla.

García-Valcárcel, A., Basilotta, V. \& López, C. (2014). Las TIC en el aprendizaje colaborativo en el aula de Primaria y Secundaria. Comunicar, 21(42), 65-74.

https://doi.org/10.3916/C42-2014-06

González, J.L., Montero, C., Batanero, M., Montero, E., de la Fuente, M.L. \& González, L. (2016). Una "inclusiva” mirada de la tecnología en nuestro colegio. Trabajando con una mirada especial: TEA y TIC en nuestro colegio. Revista Padres y Maestros, 385, 41-48.

https://doi.org/10.14422/pym.i365.y2016.006

Grande, M., Cañón, R. \& Cantón, I. (2016). Tecnologías de la Información y la Comunicación: evolución del concepto y características. International Journal of Educational Re- search and Innovation (IJERI), 6, 218-230. Recuperado de https://dialnet.unirioja.es/servlet/articulo?codigo=5732766

Guzmán, G., Putrino, N., Martínez, F. \& Quiroz, N. (2017). Nuevas tecnologías: Puentes de comunicación en el trastorno del espectro autista (TEA). Terapia Psicológica, 35(3), 247-258. https://doi.org/10.4067/S071848082017000300247

Hernández, R.M. (2017). Impacto de las TIC en la Educación: Retos y Perspectivas. Propósitos y Representaciones, 5(1), 325-347. https://doi.org/10.20511/pyr2017. v5n 1.149

Jiménez, M.D., Serrano, J.L. \& Prendes, M.P. (2017). Estudio de caso de la influencia del aprendizaje electrónico móvil en el desarrollo de la comunicación y el lenguaje con un niño con TEA. EDUCAR, 53(2), 419-443.

https://doi.org/10.5565/rev/educar.782

Kanner, L. (1943). Autistic disturbances of affective contact. Nervous child, 2, 217-250. Recuperado de http://psycnet. apa.org/record/1943-03624-001

Liu, S. H. (2011). Un modelo multivariado de factores que influyen: uso de Tecnología por futuros profesores durante la Práctica Enseñanza. Tecnología para la Educación y Sociedad, 15(4), 137-149.

Lozano, J. \& Merino, S. (2015). Utilización de las TIC’S para desarrollar las habilidades emocionales en alumnado con TEA desde la colaboración escuela-familia-universidad: una experiencia en un aula abierta específica. Revista Didáctica, Innovación y Multimedia (DIM), 31, 1-16. Recuperado de http://dimglobal.net/revistaDIM37/revistanew.htm

Lozano, J., Ballesta, F., Cerezo, M.C. \& Alcaraz, S. (2013). Las Tecnologías de la Información y la Comunicación en la relación familia-escuela. Revista Fuentes, 14, 193-208. Recuperado de https://revistascientificas.us.es/index.php/ fuentes/article/view/2359

Luján, R. (2016). Enseñanza de las TIC para el desarrollo de competencias tecnológicas en docentes de Educación Básica Alternativa. Hamut'ay, 31(1), 19-30. https://doi. org/10.21503/hamu.v3i1.997

Nicolete, P. C., Sommer, S. M., Silva, M. A., Schardosim, J. P., \& Silva, J. B. (2017). Technology Integration Actions in Mathematics teaching in Brazilian Basic Education: Stimulating STEM disciplines. Revista de Educación a Distancia (RED), 52, 1-22. https://doi.org/10.6018/red/52/7

Orozco, G.H., Tejedor, F.J. \& Calvo, M.I. (2017). Meta-Análisis sobre el efecto del Software Educativo en alumnos con Necesidades Educativas Especiales. Revista de Investigación Educativa, 35(1), 35-52. https://doi.org/10.6018/ rie.35.1.240351

Pérez, L. (2000). Aplicaciones informáticas para alumnos/as con Trastornos del Espectro Autista y otros TGD. Abriendo puertas. Congreso llevado a cabo en el X Congreso nacional de autismo, Vigo, España.

Pérez, R., Mercado, P., Martínez M., Mena E. \& Partida J.A. (2018). La sociedad del conocimiento y la sociedad de la información como la piedra angular en la innovación tecnoló- 
gica educativa. Revista Iberoamericana para la Investigación y el Desarrollo Educativo (Ride), 8(16), 847-870. https://doi.org/10.23913/ride.v8i16.371

Rodríguez de Dios, I., \& Igartua, J.J. (2015). Alfabetización digital como herramienta de protección frente a los riesgos de la red. En F. J. Durán Ruiz \& E. Said Hung (Eds.), TIC y Sociedad Digital: Educación, Infancia y Derecho (pp. 151161). Granada, España: Comares Editorial.

Rodríguez, A. \& Gallego, J.L. (2007). Socialización y edu-comunicación mediáticas para personas con deficiencias sensoriales, RIE: Revista Iberoamericana de Educación, 43(6), 1-14. Recuperado de http://www.rieoei.org/deloslectores/1810Fuentes.pdf

Rodríguez, A. \& García, A. (2012). Medios de comunicación y discapacidad. Entre la accesibilidad y la interactividad. Revista ICONO14 Revista Científica De Comunicación y Tecnologías Emergentes, 8(1), 303-319. https://doi. org/10.7195/ri14.v8i1.296

Rodríguez, A. (2007). Posibilidades y dificultades de acceso y uso del conocimiento público para personas con déficits sensoriales, EDUCAR, 39, 97-113. Recuperado de http://www.raco.cat/index.php/educar/article/viewFile/76747/99170

Rodríguez, A. (2018). Editorial. Expansión postmoderna tecnológica, escuela inclusiva tecnológica. RETOS XXI, 2, 6-12. https://doi.org/10.33412/retoxxi.v2.1.2055

Rojas, M.E. (2017). Los recursos tecnológicos como soporte para la enseñanza de las ciencias naturales. Hamut'ay, 4(1), 85-95. https://doi.org/10.21503/hamu.v4i1.1403

Romero, M. \& Harari, I. (2017). Uso de nuevas tecnologías TICS - realidad aumentada para tratamiento de niños TEA un diagnóstico inicial. CienciAmérica, 6(3), 131-137. Recuperado de https://dialnet.unirioja.es/servlet/articulo?codigo=6163694

Sánchez, J. C., Olmos, S., \& García, F. J. (2017). ¿Utilizarán los futuros docentes las tecnologías móviles? Validación de una propuesta de modelo TAM extendido. Revista de Educación a Distancia (RED), 52, 1-30.

https://doi.org/10.6018/red/52/5

Silva, G., \& Rodríguez, F. de P. (2018). Una mirada hacia las TIC en la educación de las personas con discapacidad y con Trastorno del espectro autista: Análisis temático y bibliográfico. EDMETIC, Revista de Educación Mediática y TIC, 7(1), 43-65. https://doi.org/10.21071/edmetic.v7i1.10030

Suárez, F., Mata, B. \& Peralbo, M. (2015). Valoración de un programa de intervención para niños con TEA basado en las TIC. Revista de estudios e investigación en Psicología y Educación, 9, 94-98.

https://doi.org/10.17979/reipe.2015.0.09.650

Suárez, V. (2017). El 'boom' de las Apps para personas con autismo: entre la utilidad y la fascinación. Recuperado de https://www.nobbot.com/personas/apps-autismo-utilidad-fascinacion/

Terrazas, M., Sánchez, S. \& Becerra, M.T. (2016). Las TIC como herramienta de apoyo para personas con Trastorno del
Espectro Autista (TEA). Revista nacional e internacional de educación inclusiva, 9(2), 102-136. Recuperado de https:// dialnet.unirioja.es/servlet/articulo? codigo $=5600282$

Toledo, P. \& Llorente, M.C. (2016). Formación inicial del profesorado en el uso de Tecnologías de la Información y la Comunicación (TIC) para la educación del discapacitado. Digital Education Review, 30, 135-146. Recuperado de https://dialnet.unirioja.es/servlet/articulo?codigo $=5772407$

UNESCO (2008). ICT Competency Standards for Teachers: Competency Standards Modules. Francia, París: UNESCO

Vladimirovna, S. \& Sergeevna, O. (2015). Features of the Information and Communication Technology Application by the Subjects of Special Education. International Education Studies, 8(6), 162-170.

https://doi.org/10.5539/ies.v8n6p162

Yusof, A. M., Gnanamalar, E., Daniel, S., Low, W. \& Aziz, K. (2014). Teachers' perception of mobile edutainment for special needs learners: the Malaysian case. International Journal of Inclusive Education, 18(2), 1237-1246. https://doi.org/10.1080/13603116.2014.885595

Zapata-Ros, M. (2015). Pensamiento computacional: una nueva alfabetización digital. Revista de Educación a Distancia (RED), 46(4), 1-47.

https://doi.org/10.6018/red/46/4 